\title{
A LOWER BOUND FOR A REMAINDER TERM ASSOCIATED WITH THE SUM OF DIGITS FUNCTION
}

\author{
by D. M. E. FOSTER
}

(Received 2nd May 1989)

For a fixed integer $q \geqq 2$, every positive integer $k=\sum_{r \geqq 0} a_{r}(q, k) q^{\prime}$ where each $a_{r}(q, k) \in\{0,1,2, \ldots, q-1\}$. The sum of digits function $\alpha(q, k)=\sum_{r \geq 0} a_{r}(q, k)$ behaves rather erratically but on averaging has a uniform behaviour. In particular if $A(q, n)=\sum_{k=1}^{n-1} \alpha(q, k)$, where $n>1$, then it is well known that $A(q, n) \sim$ $\frac{1}{2}((q-1) / \log q) n \log n$ as $n \rightarrow \infty$. For odd values of $q$, a lower bound is now obtained for the difference $2 S(q, n)=A(q, n)-\frac{1}{2}(q-1)[\log n / \log q] n$, where $[\log n / \log q]$ denotes the greatest integer $\leqq \log n / \log q$. This complements an upper bound already found.

1980 Mathematics subject classification (1985 Revision): 11A

\section{Introduction}

If $q \geqq 2$ is a fixed integer it is well known that every positive integer $k$ may be expressed uniquely in the form

$$
k=\sum_{r=0}^{\infty} a_{r}(q, k) q^{r} \text { where } a_{r}(q, k) \in\{0,1, \ldots, q-1\}
$$

and the "sum of digits" function $\alpha(q, k)$ is given by

$$
\alpha(q, k)=\sum_{r=0}^{\infty} a_{r}(q, k)
$$

both the above sums being finite. It is not difficult to see that, although the behaviour of $\alpha(q, k)$ itself is somewhat erratic, its average behaviour is more regular and has been widely studied.

For an integer $n>1$, let

$$
A(q, n)=\sum_{k=1}^{n-1} \alpha(q, k)
$$

and define $A(q, 1)=0$. Behaviour in the special case $n=q^{s}$ suggests the asymptotic result

$$
A(q, n) \sim \frac{\frac{1}{2}(q-1)}{\log q} n \log n \text { as } n \rightarrow \infty,
$$


a result obtained by Bush [2] in 1940. Later work by Bellman and Shapiro [1], Mirsky [11] and Drazin and Griffiths [6] gave estimates for the remainder term

$$
A(q, n)-\frac{\frac{1}{2}(q-1)}{\log q} n \log n .
$$

The case $q=2$ in particular has yielded the most precise results. In this case (and also for $q=3$ ) the results of Drazin and Griffiths are best possible and they have also been obtained by McIlroy [10] and Shiokawa [12].

In 1975, Delange [5] obtained a very elegant analytical form for the remainder term, involving a periodic, continuous but nowhere differentiable function, thereby generalizing an earlier result concerned with the case $q=2$ of Trollope [14]. In 1977, Stolarsky [13] considered the average of a more general sum of the type

$$
A_{d}(q, n)=\sum_{k=1}^{n-1}\{\alpha(q, k)\}^{d}
$$

when $q=2$ and $d \geqq 0$. (Stolarsky's paper also contains an excellent account of the history of related problems). More recently, Coquet [3] has obtained some very precise estimates for $A_{d}(q, n)$ using probabilistic techniques.

In the mid 1960s, Trollope [15] also considered the related problem concerned with Cantor representations of integers, and Kirschenhofer and Tichy [8] have since generalised Delange's result, mentioned above, to this situation too. The appropriate remainder term now takes the slightly different form

$$
S(q, n)=\left\{A(q, n)-\frac{1}{2}(q-1)\left[\frac{\log n}{\log q}\right] n\right\} / \frac{1}{2}
$$

in the special case when the Cantor representation of an integer $k$ becomes a $q$-adic representation of the form (1.1) for some $q$. As usual, $[\log n / \log q]$ denotes the greatest integer less than or equal to $\log n / \log q$. (A sum of this type has very recently been considered by Larcher and Tichy [9] in connection with the Gray code number system.) Thus, in the original digits problem, one can consider directly an estimate for $S(q, n) / n$. The best possible upper bound for all $q \geqq 2$ is $q-1$; see [7]. For $q=2,3,4,5$ and 7 the best possible lower bounds are $-2 / 3,-2 / 7,-9 / 23,-7 / 13$ and $-6 / 19$ respectively. The proofs for $q=2$ and 3 are contained in [7]. Each of these inequalities is deduced from a more precise result.

Clearly every positive integer $n \neq 0(\bmod q)$ is of the form $n=n_{m}$ where

$$
n_{m}=a_{0} q^{t_{0}}+a_{1} q^{t_{0}+t_{1}}+a_{2} q^{t_{0}+t_{1}+t_{2}}+\cdots+a_{m} q^{t_{0}+t_{1}+t_{2}+\cdots+t_{m}},
$$

for some $m \in \mathbf{N} \cup\{0\}, t_{0}=0$, positive integers $t_{1}, t_{2}, \ldots, t_{m}$ and non-zero coefficients 
$a_{0}, a_{1}, \ldots, a_{m} \in\{1,2, \ldots, q-1\}$. For convenience of notation, given such an integer $n$ introduce

$$
n_{-1}=0, n_{0}=a_{0} \quad \text { and } n_{i}=a_{0}+a_{1} q^{t_{1}}+\cdots+a_{i} q^{t_{1}+\cdots+t_{i}}
$$

for $1 \leqq i \leqq m$. Then it is not difficult to see that $S\left(q, n_{m}\right)$ has the following simple form:

$$
S\left(q, n_{m}\right)=\sum_{r=0}^{m} a_{r}\left(a_{r}-1\right) q^{t_{0}+t_{1}+\cdots+t_{r}}+\sum_{r=0}^{m}\left\{2 a_{r}-(q-1) t_{r}\right\} n_{r-1} .
$$

It is easily verified that, if $s \in \mathbf{N}$,

$$
\frac{S\left(q, q^{s} n_{m}\right)}{q^{s} n_{m}}=\frac{S\left(q, n_{m}\right)}{n_{m}}
$$

so that we may assume that $n$ is of the form (1.3) for some integer $m \geqq 0$. In [7] it was proved that

$$
\frac{S\left(q, n_{m}\right)}{n_{m}} \leqq(q-1)\left\{1-\frac{m+1}{q^{m+1}-1}\right\}
$$

with equality when $n_{m}=(q-1)\left(1+q+q^{2}+\cdots+q^{m}\right)$, together with a similar precise result for the lower bound when $q=2$ and 3 and subsequently (but not published) for $q=4,5$ and 7. However the details are rather complicated, slightly more so when $q$ is even, and the object of the present paper is to obtain an asymptotic result for all odd values of $q \geqq 9$. Numerical evidence for $5 \leqq q \leqq 13$ obtained by my colleague Mrs M. F. McCall has suggested that the likely critical case for odd $q$ occurs when $n_{m}$ is of the form

$$
n_{m}^{*}=n_{m-l}+\frac{1}{2}(q-1)\left(q^{m-1+1}+q^{m-l+2}+\cdots+q^{m-2}\right)+\frac{1}{2}\left(q-\beta_{q}\right) q^{m-1}+q^{m},
$$

where

$$
n_{m-1}=\sum_{r=0}^{m-1} a_{r} q^{t_{0}+\cdots+t_{r}}
$$

and $\beta_{q}$ is the unique odd integer satisfying

$$
3 q-\sqrt{8 q^{2}-9 q+1} \leqq \beta_{q} \leqq 3 q-\sqrt{8 q^{2}-9 q+1}+2,
$$

unless the even integer $8 q^{2}-9 q+1$ is a perfect square. If we introduce

$$
h_{q}=\frac{q^{2}-3 q-\left(\beta_{q}-1\right)\left(\beta_{q}-5\right)}{2\left(3 q-\beta_{q}+1\right)}
$$

then, as we shall see later, if $m-l$ remains fixed 


$$
\frac{S\left(q, n_{m}^{*}\right)}{n_{m}^{*}} \rightarrow-h_{q}
$$

as $m \rightarrow \infty$.

Theorem. For all odd $q \geqq 9$,

$$
\frac{S\left(q, n_{m}\right)}{n_{m}}>-h_{q}
$$

The sequence of odd integers $\left(\beta_{q}\right)$ increases with $q$ and starts off as follows:

$$
\beta_{q}=\left\{\begin{array}{lll}
3 & \text { if } q=3,5,7, \\
5 & \text { if } q=9,11, \ldots, 19 \\
7 & \text { if } q=21,23, \ldots, 31 \\
9 & \text { if } q=33,35, \ldots, 43
\end{array}\right.
$$

When $q=3,5$ or 7 , as mentioned earlier, there is a more precise result, namely

$$
\frac{S\left(q, n_{m}\right)}{n_{m}} \geqq-h_{q}(m)
$$

where

$$
h_{3}(m)=\frac{6\left(3^{m}-1\right)}{7.3^{m+1}-5} \quad \text { and } \quad h_{q}(m)=\frac{\left(q^{2}-3 q+4\right) q^{m-1}-1}{2\left\{(3 q-2) q^{m-1}-1\right\}} \quad(q=5,7)
$$

In each of these cases $h_{q}(m) \uparrow h_{q}$ as $m \uparrow \infty$.

In the special case when $8 q^{2}-9 q+1=(2 x)^{2}$ for some positive integer $x$, it may be verified that either choice of $\beta_{q}=3 q-2 x$ or $3 q-2 x+2$ in (1.5) gives rise to the same value of $h_{q}$ in (1.6). This case can only arise when $q \equiv 1(\bmod 4)$, so that we can express $q=1+4 y$ where $y(32 y+7)=x^{2}$. Let $d=h c f(x, y)$ with $x=x_{1} d$ and $y=y_{1} d$ for positive coprime integers $x_{1}$ and $y_{1}$. Then $y_{1}\left(32 d y_{1}+7\right)=d x_{1}^{2}$ giving $d=y_{1} y_{2}$ for some integer $y_{2}$. This leads to $y_{2}\left(x_{1}^{2}-32 y_{1}^{2}\right)=7$ so that $y_{2}=1$ or 7 . If $y_{2}=1, x_{1}^{2}-32 y_{1}^{2}=7$ which is insoluble since $x_{1}$ must be odd giving $x_{1}^{2} \equiv 1(\bmod 4)$. Thus $y_{2}=7$ and we are left with the Pell equation $x_{1}^{2}-32 y_{1}^{2}=1$ whose general solution for positive integers $x_{1}$ and $y_{1}$ is given by

$$
x_{1}+\sqrt{32} y_{1}=(17+3 \sqrt{32})^{r}, \quad r \in \mathbf{N} \text {. }
$$

This solution arises from the continued fraction expression of $\sqrt{ } 32$. (See, for example, 
Davenport [4]). The two smallest values of $q$ which occur are $253(r=1)$ and 291313 $(r=2)$; and when $q=253$ it is easily verified that $\beta_{q}=45$ or 47 .

It is convenient to prove the theorem in the following equivalent form. Introduce

$$
\alpha_{q}=h_{q}-1 \text { so that } \alpha_{q}=\frac{q^{2}-9 q-\left(\beta_{q}-1\right)\left(\beta_{q}-7\right)}{2\left(3 q-\beta_{q}+1\right)} .
$$

Then

$$
\left(\alpha_{9}, \alpha_{11}, \alpha_{13}, \alpha_{15}, \alpha_{17}, \alpha_{19}\right)=\left(\frac{4}{23}, \frac{15}{29}, \frac{6}{7}, \frac{49}{41}, \frac{72}{47}, \frac{99}{53}\right)
$$

and

$$
\left(\alpha_{21}, \alpha_{23}, \alpha_{25}, \alpha_{27}, \alpha_{29} \alpha_{31}\right)=\left(\frac{42}{19}, \frac{23}{9}, \frac{200}{69}, \frac{81}{25}, \frac{290}{81}, \frac{341}{87}\right),
$$

and so on. For $m \geqq 0$ we define

$$
\begin{aligned}
T\left(q, n_{m}\right)= & S\left(q, n_{m}\right)+h_{q} n_{m} \\
= & S\left(q, n_{m}\right)+\left(\alpha_{q}+1\right) n_{m} \\
= & \sum_{r=0}^{m} a_{r}\left(a_{r}+\alpha_{q}\right) q^{t_{0}+t_{1}+\cdots+t_{r}} \\
& +\sum_{r=0}^{m}\left\{2 a_{r}-(q-1) t_{r}\right\} n_{r-1} .
\end{aligned}
$$

Then the inequality of the theorem is equivalent to

$$
T\left(q, n_{m}\right)>0 .
$$

I am very grateful to Mrs McCall for her help in giving me a feel for the general result. I should also like to thank the referee for his very careful corrections of many errors in the original draft.

\section{2.}

In this section we prove three lemmas which will be needed in the proof of the theorem.

Lemma 2.1. $3 \leqq \beta_{q}-\alpha_{q} \leqq 5$. 
Proof. We have, using (1.7) and rearranging,

$$
\beta_{q}-\alpha_{q}-3=\frac{8 q^{2}-9 q+1-\left(\beta_{q}-3 q\right)^{2}}{2\left(3 q-\beta_{q}+1\right)} \geqq 0, \quad \text { using (1.5). }
$$

In fact, if $8 q^{2}-9 q+1$ is not a perfect square, then as both $8 q^{2}-9 q+1$ and $\beta_{q}-3 q$ are even integers it is clear that

$$
\beta_{q}-\alpha_{q}-3 \geqq \frac{1}{3 q-\beta_{q}+1}>\frac{1}{3 q},
$$

a result which will be of use later.

Similarly,

$$
5-\left(\beta_{q}-\alpha_{q}\right)=\frac{\left(3 q-\beta_{q}+2\right)^{2}-\left(8 q^{2}-9 q+1\right)}{2\left(3 q-\beta_{q}+1\right)} \geqq 0 \quad \text { using (1.5). }
$$

Once again, if $8 q^{2}-9 q+1$ is not a perfect square then

$$
5-\left(\beta_{q}-\alpha_{q}\right)>\frac{1}{3 q} \text {. }
$$

Lemma 2.2. Let $e$ and $f$ be real numbers and let $x_{0}$ be a positive integer with $(q-1) x_{0}-e \geqq 1$. Then, if the inequality

$$
4\{(q-1) x-e\} \leqq \int q^{x-1}
$$

holds for $x=x_{0}$, it also holds for all integers $x>x_{0}$.

Proof. Clearly $f q^{x_{0}-1} \geqq 4$. If we take $x=x_{0}+1$ we have

$$
\begin{aligned}
4\left\{(q-1)\left(x_{0}+1\right)-e\right\} & =4\left\{(q-1) x_{0}-e\right\}+4(q-1) \\
& \leqq f q^{x_{0}-1}+4(q-1) \\
& \leqq f q^{x_{0}}
\end{aligned}
$$

provided that

$$
4(q-1) \leqq f q^{x_{0}-1}(q-1)
$$

which is true.

It is convenient at this point to introduce some useful substitutions for $a_{m-1}, a_{m-2}, \ldots$ chosen to reflect the critical case when $n=n_{m}^{*}$. These take the form

$$
2 a_{m-1}=q-\beta_{q}+2 \delta_{1}
$$


and

$$
2 a_{r}=q-1-2 \delta_{m-r-1}+2 \delta_{m-r}(m-2 \geqq r \geqq 0)
$$

where $\delta_{1}, \delta_{2}, \ldots, \delta_{m} \in \mathbf{Z}$.

The final lemma facilitates some routine calculations which are repeated throughout the proof of the theorem at all the different stages.

If $l$ is any integer satisfying $2 \leqq l \leqq m+1$, for convenience of notation introduce

$$
F_{m}(l-1)=a_{m}\left(a_{m}+\alpha_{q}\right) q^{t_{1}+\cdots+t_{m}}+\sum_{r=m-l+1}^{m-1} a_{r}\left\{\sum_{s=r+1}^{m}\left[2 a_{s}-(q-1) t_{s}\right]+a_{r}+\alpha_{q}\right\} q^{i_{1}+\cdots+t_{r}}
$$

It is useful to rearrange $F_{m}(l-1)$ as follows. We have

$$
F_{m}(l-1)=\sum_{r=m-l+1}^{m} a_{r}\left(a_{r}+\alpha_{q}\right) q^{t_{1}+\cdots+t_{r}}+\sum_{r=m-l+1}^{m-1} a_{r}\left\{\sum_{s=r+1}^{m}\left[2 a_{s}-(q-1) t_{s}\right]\right\} q^{t_{1}+\cdots+t_{r}},
$$

where the double sum may be expressed as

$$
\begin{aligned}
& \sum_{s=m-l+2}^{m}\left[2 a_{s}-(q-1) t_{s}\right] \sum_{r=m-l+1}^{s-1} a_{r} q^{t_{1}+\cdots+t_{r}} \\
& =\sum_{s=m-l+2}^{m}\left[2 a_{s}-(q-1) t_{s}\right] n_{s-1}-n_{m-1} \sum_{s=m-l+2}^{m}\left[2 a_{s}-(q-1) t_{s}\right] .
\end{aligned}
$$

Thus

$$
\begin{aligned}
F_{m}(l-1)= & \sum_{r=m-l+1}^{m}\left\{a_{r}\left(a_{r}+\alpha_{q}\right) q^{t_{1}+\cdots+t_{r}}+\left[2 a_{r}-(q-1) t_{r}\right] n_{r-1}\right\} \\
& -n_{m-l} \sum_{r=m-l+1}^{m}\left[2 a_{r}-(q-1) t_{r}\right] .
\end{aligned}
$$

As $n_{-1}=0$, putting $l=m+1$ we have in particular

$$
F_{m}(m)=T\left(q, n_{m}\right)
$$

Lemma 2.3. If $2 \leqq l \leqq m+1, \quad t_{m}=t_{m-1}=\cdots=t_{m-1+2}=1, \quad a_{m}=1$ and $a_{m-1}$, $a_{m-2}, \ldots, a_{m-1+1}$ are given by (2.4) then

$$
4 F_{m}(l-1) q^{-\left(t_{1}+\cdots+t_{m-1+1}\right)}=4(q-1) \sum_{s=1}^{l-2} q^{l-2-s} k\left(\delta_{s}\right)+4 h\left(\delta_{l-1}\right)+q-7-2 \alpha_{q}+2 \beta_{q},
$$

where

$$
h(x)=x\left(x+3+\alpha_{q}-\beta_{q}\right)
$$


and

$$
k(x)=x\left(x+4+\alpha_{q}-\beta_{q}\right) .
$$

In the particular case when $l=2$ the summation

$$
\sum_{s=1}^{l-2}
$$

is assumed to be vacuous.

Proof. When $l=2$ we have

$$
F_{m}(1)=a_{m}\left(a_{m}+\alpha_{q}\right) q^{t_{1}+\cdots+t_{m}}+a_{m-1}\left\{2 a_{m}-(q-1) t_{m}+a_{m-1}+\alpha_{q}\right\} q^{t_{1}+\cdots+t_{m-1}},
$$

together with $t_{m}=a_{m}=1$ and $2 a_{m-1}=q-\beta_{q}+2 \delta_{1}$. Thus

$$
\begin{aligned}
4 F_{m}(1) q_{q}^{-\left(t_{1}+\cdots+t_{m-1}\right)} & =4\left(1+\alpha_{q}\right) q-\left(q-\beta_{q}+2 \delta_{1}\right)\left(q+\beta_{q}-2 \delta_{1}-6-2 \alpha_{q}\right) \\
& =4 \delta_{1}\left(\delta_{1}+3+\alpha_{q}-\beta_{q}\right)+10 q-q^{2}+\beta_{q}^{2}-6 \beta_{q}-2 \alpha_{q}+2 \alpha_{q}\left(3 q-\beta_{q}+1\right) .
\end{aligned}
$$

Using (1.7) to eliminate $\alpha_{q}$ from the last term we eventually obtain

$$
4 F_{m}(1) q^{-\left(i_{1}+\cdots+t_{m-1}\right)}=4 h\left(\delta_{1}\right)+q-7-2 \alpha_{q}+2 \beta_{q} .
$$

When $l=3$, we have

$$
F_{m}(2)=F_{m}(1)+a_{m-2}\left\{2\left(a_{m}+a_{m-1}\right)-(q-1)\left(t_{m}+t_{m-1}\right)+a_{m-2}+\alpha_{q}\right\} q^{t_{1}+\cdots+t_{m-2}},
$$

together with $t_{m}=t_{m-1}=a_{m}=1$,

$$
2 a_{m-1}=q-\beta_{q}+2 \delta_{1} \text { and } 2 a_{m-2}=q-1-2 \delta_{1}+2 \delta_{2} .
$$

Using the above simplification of $F_{m}(1)$ we see that

$$
4 F_{m}(2) q^{-\left(t_{1}+\cdots+t_{m-2}\right)}=q\left\{4 h\left(\delta_{1}\right)+q-7-2 \alpha_{q}+2 \beta_{q}\right\}+4 a_{m-2}\left(a_{m-2}-q+4+\alpha_{q}-\beta_{q}+2 \delta_{1}\right) .
$$

The terms involving $a_{m-2}$ are

$$
\begin{aligned}
& \left(q-1-2 \delta_{1}+2 \delta_{2}\right)\left(-q-1+2 \delta_{1}+2 \delta_{2}+8+2 \alpha_{q}-2 \beta_{q}\right) \\
& =-\left(q-2 \delta_{1}\right)^{2}+\left(2 \delta_{2}-1\right)^{2}+\left(q-1-2 \delta_{1}+2 \delta_{2}\right)\left(8+2 \alpha_{q}-2 \beta_{q}\right) \\
& =-(q-1)\left(q-7-2 \alpha_{q}+2 \beta_{q}\right)+4(q-1) \delta_{1}-4 h\left(\delta_{1}\right)+4 h\left(\delta_{2}\right),
\end{aligned}
$$

following rearrangement. Thus

$$
\begin{aligned}
& 4 F_{m}(2) q^{-\left(t_{1}+\cdots+t_{m-2}\right)} \\
& \quad=4 q h\left(\delta_{1}\right)+q\left(q-7-2 \alpha_{q}+2 \beta_{q}\right)-(q-1)\left(q-7-2 \alpha_{q}+2 \beta_{q}\right)+4(q-1) \delta_{1}-4 h\left(\delta_{1}\right)+4 h\left(\delta_{2}\right),
\end{aligned}
$$

and this takes the required form 


$$
4 F_{m}(2) q^{-\left(t_{1}+\cdots+t_{m}-2\right)}=4(q-1) k\left(\delta_{1}\right)+4 h\left(\delta_{2}\right)+q-7-2 \alpha_{q}+2 \beta_{q} .
$$

The general case is now quite easy. Assume that (2.7) holds for some integer 1 satisfying $2 \leqq l \leqq m$ and look at $F_{m}(l)$. We have

$$
F_{m}(l)=F_{m}(l-1)+a_{m-l}\left\{\sum_{s=m-l+1}^{m}\left[2 a_{s}-(q-1) t_{s}\right]+a_{m-1}+\alpha_{q}\right\} q^{t_{1}+\cdots+t_{m-1}} .
$$

By the hypothesis of this case, we have

$$
t_{m}=t_{m-1}=\cdots=t_{m-1+1}=1 \text { and } a_{m}=1
$$

together with the usual substitutions for $a_{m-1}, \ldots, a_{m-l}$. Thus on substituting we obtain

$$
F_{m}(l)=F_{m}(l-1)+a_{m-l}\left(a_{m-l}-q+4+\alpha_{q}-\beta_{q}+2 \delta_{l-1}\right) q^{i_{1}+\cdots+t_{m-1}} .
$$

Using (2.7) we see that

$$
\begin{aligned}
4 q^{-\left(t_{1}+\cdots+t_{m-1}\right)} F_{m}(l)= & 4(q-1) \sum_{s=1}^{t-2} q^{l-1-s} k\left(\delta_{s}\right) \\
& +4 q h\left(\delta_{l-1}\right)+q\left(q-7-2 \alpha_{q}+2 \beta_{q}\right) \\
& +4 a_{m-l}\left(a_{m-l}-q+4+\alpha_{q}-\beta_{q}+2 \delta_{l-1}\right)
\end{aligned}
$$

Since $2 a_{m-l}=q-1-2 \delta_{l-1}+2 \delta_{l}$ we have a similar calculation as in the evaluation of $F_{m}(2)$, with an obvious change of notation. This leads to

$$
4 q^{-\left(t_{1}+\cdots+t_{m-l)}\right.} F_{m}(l)=4(q-1) \sum_{s=1}^{l-2} q^{l-1-s} k\left(\delta_{s}\right)+4(q-1) k\left(\delta_{l-1}\right)+4 h\left(\delta_{l}\right)+q-7-2 \alpha_{q}+2 \beta_{q},
$$

and this is (2.7) with $l$ replaced by $l+1$.

\section{Proof of the theorem}

We proceed by induction on the integer $m \geqq 0$. Initially

$$
T\left(q, n_{0}\right)=a_{0}\left(a_{0}+\alpha_{q}\right)>0,
$$

since $a_{0} \geqq 1$ and $\alpha_{q}>0 \forall q \geqq 9$. Thus we now choose $m \geqq 1$ and assume that

$$
T\left(q, n_{r}\right)>0 \forall 0 \leqq r \leqq m-1 .
$$

The proof runs through several stages, ending up with the main inductive step once the critical form for $n_{m}$ begins to emerge. 
Stage $1(m \geqq 1)$. We can express

$$
T\left(q, n_{m}\right)=a_{m}\left(a_{m}+\alpha_{q}\right) q^{i_{1}+\cdots+t_{m}}+\left\{2 a_{m}-(q-1) t_{m}\right\} n_{m-1}+T\left(q, n_{m-1}\right) .
$$

By the induction hypothesis (3.1), $T\left(q, n_{m-1}\right)>0$ and it will therefore follow that $T\left(q, n_{m}\right)>0$ provided that

$$
\left\{(q-1) t_{m}-2 a_{m}\right\} n_{m-1}<a_{m}\left(a_{m}+\alpha_{q}\right) q^{i_{1}+\cdots+t_{m}} .
$$

If $2 a_{m} \geqq(q-1) t_{m},(3.2)$ follows easily. Thus suppose that $(q-1) t_{m}>2 a_{m}$. Since

$$
\begin{aligned}
n_{m-1} & =a_{0}+a_{1} q^{t_{1}}+a_{2} q^{t_{1}+t_{2}}+\cdots+a_{m-1} q^{t_{1}+t_{2} \cdots+t_{m-1}} \\
& \leqq(q-1)\left\{1+q^{t_{1}}+q^{t_{1}+t_{2}}+\cdots+q^{t_{1}+t_{2}+\cdots+t_{m-1}}\right\} \\
& \leqq q^{t_{1}+t_{2}+\cdots+t_{m-1}+1}-1<q^{t_{1}+t_{2}+\cdots+t_{m-1}+1},
\end{aligned}
$$

the inequality (3.2) will hold provided that

$$
(q-1) t_{m}-2 a_{m}<a_{m}\left(a_{m}+\alpha_{q}\right) q^{i_{m}-1} .
$$

If $t_{m}=2,(3.3)$ takes the form

$$
2(q-1)<a_{m}\left\{\left(a_{m}+\alpha_{q}\right) q+2\right\}
$$

which holds easily $\forall a_{m} \geqq 2$. When $a_{m}=1$, the inequality becomes $\left(\alpha_{q}-1\right) q+4>0$. For $q \geqq 15, \alpha_{q}>1$ and for $9 \leqq q \leqq 13, \alpha_{q}<1$ and the inequality is equivalent to $q \alpha_{q}>q-4$ which holds for $q=13$. Thus we are left only with the cases $q=9$ and 11 .

If $t_{m}=3,(3.3)$ becomes

$$
3(q-1) \leqq a_{m}\left\{\left(a_{m}+\alpha_{q}\right) q^{2}+2\right\}
$$

which holds for all $a_{m} \geqq 1$ and $q \geqq 9$. As $(q-1) t_{m}-2 a_{m} \geqq 1$ we can now appeal to Lemma 2.2 to deduce that (3.3) holds for all integers $t_{m} \geqq 3$ when $q \geqq 9$.

Thus we have proved that $T\left(q, n_{m}\right)>0$ except in the following cases:

$$
t_{m}=1
$$

or

$$
\left(t_{m} ; a_{m}\right)=(2 ; 1) \text { when } q=9 \text { or } 11
$$

If $m=1$, we have

$$
T\left(q, n_{1}\right)=a_{1}\left(a_{1}+\alpha_{q}\right) q^{t_{1}}+a_{0}\left\{a_{0}+\alpha_{q}+2 a_{1}-(q-1) t_{1}\right\} .
$$

The only cases which we need consider are I(i) and I(ii). In the first case, $t_{1}=1$ and $a_{1} \geqq 1$ so that 


$$
T\left(q, n_{1}\right) \geqq\left(1+\alpha_{q}\right) q+a_{0}\left(a_{0}-q+3+\alpha_{q}\right) .
$$

We now make the usual substitution for $a_{0}$ namely

$$
2 a_{0}=2 a_{m-1}=q-\beta_{q}+2 \delta_{1},
$$

and using the case $l=2$ of Lemma 2.3 we see that

$$
4 T\left(q, n_{1}\right) \geqq 4 h\left(\delta_{1}\right)+q-7-2 \alpha_{q}+2 \beta_{q} .
$$

By Lemma $2.1,3 \leqq \beta_{q}-\alpha_{q} \leqq 5$ so that $q-7-2 \alpha_{q}+2 \beta_{q} \geqq q-1$. Also, for integral values of $\delta_{1}$ the two least values of $h\left(\delta_{1}\right)$ occur when $\delta_{1}=0$ and 1 . Clearly $h(0)=0$ and $h(1)=4+\alpha_{q}-\beta_{q} \geqq-1$. Thus in the worst case

$$
4 T\left(q, n_{1}\right) \geqq-4+q-1=q-5>0 .
$$

In the remaining case I(ii) when $q=9$ or 11 we have $\left(t_{m} ; a_{m}\right)=\left(t_{1} ; a_{1}\right)=(2 ; 1)$. Hence

$$
T\left(q, n_{1}\right)=\left(1+\alpha_{q}\right) q^{2}+a_{0}\left(a_{0}-2 q+4+\alpha_{q}\right) \text {. }
$$

Directly,

$$
T\left(9, n_{1}\right)=81\left(1+\alpha_{9}\right)+a_{0}\left(a_{0}+\alpha_{9}-14\right)
$$

and

$$
T\left(11, n_{1}\right)=121\left(1+\alpha_{11}\right)+a_{0}\left(a_{0}+\alpha_{11}-18\right),
$$

with $\alpha_{9}=4 / 23$ and $\alpha_{11}=15 / 29$ and in each case $T\left(q, n_{1}\right)>0 \forall a_{0} \geqq 1$.

We can now assume that $m \geqq 2$ and proceed to the next stage of the argument.

Stage $2(m \geqq 2)$. We subdivide $T\left(q, n_{m}\right)$ further as follows:

$$
\begin{aligned}
T\left(q, n_{m}\right)= & \sum_{r=m-1}^{m} a_{r}\left(a_{r}+\alpha_{q}\right) q^{t_{1}+\cdots+t_{r}} \\
& +\left\{2 a_{m}-(q-1) t_{m}\right\}\left(n_{m-2}+a_{m-1} q^{t_{1}+\cdots+t_{m-1}}\right) \\
& +\left\{2 a_{m-1}-(q-1) t_{m-1}\right\} n_{m-2}+T\left(q, n_{m-2}\right)
\end{aligned}
$$

By (3.1), $T\left(q, n_{m-2}\right)>0$ and so $T\left(q, n_{m}\right)>0$ provided that

$$
\begin{aligned}
& \left\{(q-1)\left(t_{m}+t_{m-1}\right)-2\left(a_{m}+a_{m-1}\right)\right\} n_{m-2} \\
& \quad \leqq q^{t_{1}+\cdots+t_{m-1}}\left[a_{m}\left(a_{m}+\alpha_{q}\right) q^{t_{m}}+a_{m-1}\left\{2 a_{m}-(q-1) t_{m}+a_{m-1}+\alpha_{q}\right\}\right] .
\end{aligned}
$$

If $(q-1)\left(t_{m}+t_{m-1}\right)-2\left(a_{m}+a_{m-1}\right) \leqq 0$, the left-hand side of $(3.5)$ is non-positive and the right-hand side is positive since 


$$
2 a_{m}-(q-1) t_{m}+a_{m-1}+\alpha_{q} \geqq(q-1) t_{m-1}-a_{m-1}+\alpha_{q} \geqq q-1-a_{m-1}+\alpha_{q}
$$

and $\alpha_{q}>0 \forall q \geqq 9$. Thus we shall assume henceforth that

$$
(q-1)\left(t_{m}+t_{m-1}\right)-2\left(a_{m}+a_{m-1}\right)>0 .
$$

As

$$
\begin{aligned}
n_{m-2} & \leqq(q-1)\left(1+q^{t_{1}}+q^{t_{1}+t_{2}}+\cdots+q^{t_{1}+t_{2}+\cdots+t_{m-2}}\right) \\
& \leqq q^{t_{1}+t_{2}+\cdots+t_{m-2}+1}-1<q^{t_{1}+t_{2}+\cdots t_{m-2}+1},
\end{aligned}
$$

it will follow that $T\left(q, n_{m}\right)>0$ provided that

$$
\begin{aligned}
& q^{-\left(t_{m-1}-1\right)}\left\{(q-1)\left(t_{m}+t_{m-1}\right)-2\left(a_{m}+a_{m-1}\right)\right\} \\
& \quad \leqq a_{m}\left(a_{m}+\alpha_{q}\right) q^{t_{m}}+a_{m-1}\left\{2 a_{m}-(q-1) t_{m}+a_{m-1}+\alpha_{q}\right\} .
\end{aligned}
$$

Case I(i). Putting $t_{m}=1$, (3.7) takes the form

$$
\begin{aligned}
& q^{-\left(t_{m-1}-1\right)}\left\{(q-1) t_{m-1}-2\left(a_{m}+a_{m-1}\right)+q-1\right\} \\
& \quad \leqq a_{m}\left(a_{m}+\alpha_{q}\right) q+a_{m-1}\left(a_{m-1}+2 a_{m}-q+1+\alpha_{q}\right) .
\end{aligned}
$$

When $a_{m}=2$, we shall prove that (3.8) holds for all integers $1 \leqq a_{m-1} \leqq q-1$ and $t_{m-1} \geqq 1$. Since the coefficient of $a_{m}$ on the right-hand side of (3.8) is positive and that on the left-hand side is negative, the inequality will then clearly follow for all integers $a_{m} \geqq 2$. But first we make the usual substitution $2 a_{m-1}=q-\beta_{q}+2 \delta_{1}$ and then, after some rearrangement, (3.8) becomes

$$
\begin{aligned}
& 4 q^{-\left(t_{m-1}-1\right)}\left\{(q-1) t_{m-1}-2 a_{m}+\beta_{q}-2 \delta_{1}-1\right\} \\
& \leqq \\
& \quad 4 \delta_{1}\left(\delta_{1}+2 a_{m}+1+\alpha_{q}-\beta_{q}\right)+2 q-q^{2}+\beta_{q}^{2}-2 \beta_{q}+4 a_{m}\left\{\left(a_{m}+1\right) q-\beta_{q}\right\} \\
& \quad+2 \alpha_{q}\left\{2\left(a_{m}-1\right) q-1\right\}+2 \alpha_{q}\left(3 q-\beta_{q}+1\right) .
\end{aligned}
$$

Using (1.7) to eliminate $\alpha_{q}$ from the last term on the right-hand side we obtain the following inequality:

$$
\begin{aligned}
& 4 q^{-\left(t_{m-1}-1\right)}\left\{(q-1) t_{m-1}-2 a_{m}+\beta_{q}-2 \delta_{1}-1\right\} \\
& \leqq \\
& \quad 4 \delta_{1}\left(\delta_{1}+2 a_{m}+1+\alpha_{q}-\beta_{q}\right)+4 a_{m}\left\{\left(a_{m}+1\right) q-\beta_{q}\right\} \\
& \quad+4\left(a_{m}-1\right) \alpha_{q} q-7 q-7-2 \alpha_{q}+6 \beta_{q} .
\end{aligned}
$$

Putting $a_{m}=2$ as indicated earlier, (3.9) becomes

$$
4 q^{-\left(t_{m-1}-1\right)}\left\{(q-1) t_{m-1}-5+\beta_{q}-2 \delta_{1}\right\}
$$




$$
\leqq 4 \delta_{1}\left(\delta_{1}+5+\alpha_{q}-\beta_{q}\right)+17 q-7+2 \alpha_{q}(2 q-1)-2 \beta_{q} .
$$

By (3.6) the integer $(q-1) t_{m-1}-5+\beta_{q}-2 \delta_{1}>0$, and consequently the validity of (3.10) for $t_{m-1}=1$ will follow for all integers $t_{m-1}>1$ by Lemma 2.2. Putting $t_{m-1}=1$ in (3.10) leads to the inequality

$$
4 \delta_{1}\left(\delta_{1}+7+\alpha_{q}-\beta_{q}\right)+13 q+17+2(2 q-1) \alpha_{q}-6 \beta_{q} \geqq 0
$$

As $3 \leqq \beta_{q}-\alpha_{q} \leqq 5$, the least value of $\delta_{1}\left(\delta_{1}+7+\alpha_{q}-\beta_{q}\right)$ occurs when $\delta_{1}=-1$ if $4<\beta_{q}-\alpha_{q} \leqq 5$ and when $\delta_{1}=-2$ if $3 \leqq \beta_{q}-\alpha_{q} \leqq 4$. In the first case (3.11) becomes

$$
q-7+2\left(6 q-\beta_{q}\right)+2(2 q-3) \alpha_{q} \geqq 0
$$

which holds easily as $\beta_{q}<6 q$, and in the second case (3.11) becomes

$$
13 q-23+2(2 q-5) \alpha_{q}+2 \beta_{q} \geq 0
$$

which again causes no problems.

The rest of Case I(i) is concerned with verifying (3.9) when $a_{m}=1$ for all integers $t_{m-1} \geqq 2$. Once again we take $t_{m-1}=2$ and then appeal to Lemma 2.2 to obtain its validity for the integral values of $t_{m-1}>2$. First putting $a_{m}=1$ in (3.9) we have

$$
\begin{aligned}
& 4 q^{-\left(t_{m-1}-1\right)}\left\{(q-1) t_{m-1}-3+\beta_{q}-2 \delta_{1}\right\} \\
& \leqq 4 \delta_{1}\left(\delta_{1}+3+\alpha_{q}-\beta_{q}\right)+q-7-2 \alpha_{q}+2 \beta_{q} .
\end{aligned}
$$

When $t_{m-1}=2$, (3.12) rearranges to the form

$$
4 q \delta_{1}\left(\delta_{1}+3+\alpha_{q}-\beta_{q}+2 q^{-1}\right)+q^{2}-15 q+2\left(\beta_{q}-\alpha_{q}\right) q-4 \beta_{q}+20 \geqq 0 .
$$

Since $3 \leqq \beta_{q}-\alpha_{q} \leqq 5$ and $q \geqq 9$ we have

$$
-\frac{2}{9} \leqq \beta_{q}-\alpha_{q}-3-2 q^{-1}<2
$$

and it follows that the least value of $\delta_{1}\left(\delta_{1}+3+\alpha_{q}-\beta_{q}+2 q^{-1}\right)$ for integral $\delta_{1}$ occurs when $\delta_{1}=0$ or 1 . Putting $\delta_{1}=0$ we need to check the inequality

$$
q^{2}-15 q+2\left(\beta_{q}-\alpha_{q}\right) q-4 \beta_{q}+20 \geqq 0 .
$$

As $\beta_{q}-\alpha_{q} \geqq 3$ it will suffice to prove that

$$
4 \beta_{q} \leqq q(q-9)+20 .
$$

If $\beta_{q}=5$ we have $9 \leqq q \leqq 19$ and there is no problem. Thus suppose that $\beta_{q} \geqq 7$ giving $q \geqq 21$. By (1.5), $\beta_{q} \leqq 3 q-\sqrt{ }\left(8 q^{2}-9 q+1\right)+2$ and it will suffice to verify that

$$
q(q-21)+4 \sqrt{ }\left(8 q^{2}-9 q+1\right)+12 \geqq 0
$$


which is easily true $\forall q \geqq 21$. Now putting $\delta_{1}=1$ we need to check the inequality

$$
q^{2}+\left\{2\left(\alpha_{q}-\beta_{q}\right)+1\right\} q-4 \beta_{q}+28 \geqq 0 .
$$

As $\alpha_{q}-\beta_{q} \geqq-5$, it will suffice to show that

$$
4 \beta_{q} \leqq q(q-9)+28
$$

and this has already been proved with 28 replaced by 20 .

Thus in Case I(i) we are left only with $a_{m}=1$ and $t_{m-1}=1$.

Case I(ii). Putting $t_{m}=2$ and $a_{m}=1$ in (3.7) leads to the inequality

$$
(q-1) t_{m-1}-2 a_{m-1}+2 q-4 \leqq\left(1+\alpha_{q}\right) q^{t_{m-1}+1}+a_{m-1}\left(a_{m-1}-2 q+4+\alpha_{q}\right) q^{t_{m-1}-1} .
$$

For $q=9$ and $11,(3.15)$ is easily verified for all $1 \leqq a_{m-1} \leqq q-1$ when $t_{m-1}=1$. As the right-hand side increases exponentially with $t_{m-1}$, there is no problem when $t_{m-1} \geqq 2$.

Summing up, if $m \geqq 2$ we have proved the result except in the following case:

$$
\left(t_{m}, t_{m-1} ; a_{m}\right)=(1,1 ; 1)
$$

If $m=2$, by (2.6) we have $T\left(q, n_{2}\right)=F_{2}(2)$. Then, using condition II together with the usual substitutions for $a_{1}$ and $a_{0}$, we see from Lemma 2.3 that $T\left(q, n_{2}\right)>0$ is equivalent to

$$
4(q-1) k\left(\delta_{1}\right)+4 h\left(\delta_{2}\right)+q-7-2 \alpha_{q}+2 \beta_{q}>0
$$

where as usual

$$
k\left(\delta_{1}\right)=\delta_{1}\left(\delta_{1}+4+\alpha_{q}-\beta_{q}\right) \text { and } h\left(\delta_{2}\right)=\delta_{2}\left(\delta_{2}+3+\alpha_{q}-\beta_{q}\right)
$$

As $3 \leqq \beta_{q}-\alpha_{q} \leqq 5, k\left(\delta_{1}\right) \geqq k(0)=0$ for all integers $\delta_{1}$ and (3.16) is therefore a consequence of

$$
4 h\left(\delta_{2}\right)+q-7-2 \alpha_{q}+2 \beta_{q}>0
$$

an inequality which has already been seen to be true at the end of stage 1 in the case when $m=1$.

We now take $m \geqq 3$ and let $l$ be any integer satisfying $3 \leqq l \leqq m$.

Stage $l(3 \leqq l \leqq m)$. As a consequence of stages 1 and 2 we now make the inductive assumption that

$$
t_{m}=t_{m-1}=\cdots=t_{m-1+2}=1 \text { and } a_{m}=1
$$

together with the substitutions in (2.4) for $a_{m-1}, a_{m-2}, \ldots, a_{m-1+1}$. Following the customary procedure we express 
$T\left(q, n_{m}\right)=\sum_{r=m-1+1}^{m} a_{r}\left(a_{r}+\alpha_{q}\right) q^{t_{1}+\cdots+t_{r}}+\sum_{r=m-l+1}^{m}\left\{2 a_{r}-(q-1) t_{r}\right\} n_{r-1}+T\left(q, n_{m-l}\right)$.

By (3.1), $T\left(q, n_{m-1}\right)>0$ and we therefore have to prove that

$$
\begin{aligned}
& {\left[\sum_{r=m-1+1}^{m}\left\{(q-1) t_{r}-2 a_{r}\right\}\right] n_{m-1}} \\
& \quad \leqq a_{m}\left(a_{m}+\alpha_{q}\right) q^{t_{1}+\cdots+t_{m}}+\sum_{r=m-l+1}^{m-1} a_{r}\left\{\sum_{s=r+1}^{m}\left[2 a_{s}-(q-1) t_{s}\right]+a_{r}+\alpha_{q}\right\} q^{t_{1}+\cdots+t_{r}} .
\end{aligned}
$$

The expression on the right hand side of (3.18) is simply the $F_{m}(l-1)$ of Lemma 2.3, and accordingly we obtain the following simplification of (3.18), namely

$$
\begin{aligned}
& 4 q^{-\left(t_{1}+\cdots+t_{m-1+1}\right)}\left\{(q-1) t_{m-l+1}-3+\beta_{q}-2 \delta_{l-1}\right\} n_{m-l} \\
& \leqq 4(q-1) \sum_{s=1}^{l-2} q^{l-2-s} k\left(\delta_{s}\right)+4 h\left(\delta_{l-1}\right)+q-7-2 \alpha_{q}+2 \beta_{q} .
\end{aligned}
$$

For each integral value of $\delta_{s}, k\left(\delta_{s}\right) \geqq k(0)=0$ and $4 h\left(\delta_{l-1}\right)+q-7-2 \alpha_{q}+2 \beta_{q}>0$ as we have already seen. Thus for each $1 \leqq s \leqq l-2$ the right-hand side of (3.19) is positive. Hence it will suffice to consider the case when

$$
(q-1) t_{m-1+1}-3+\beta_{q}-2 \delta_{l-1}>0 .
$$

As $n_{m-1}<q^{t_{1}+\cdots+t_{m-1}+1},(3.19)$ is a consequence of

$$
\begin{aligned}
& 4 q^{-\left(t_{m-1+1}-1\right)}\left\{(q-1) t_{m-l+1}-3+\beta_{q}-2 \delta_{l-1}\right\} \\
& \leqq 4(q-1) \sum_{s=1}^{l-2} q^{l-2-s} k\left(\delta_{s}\right)+4 h\left(\delta_{l-1}\right)+q-7-2 \alpha_{q}+2 \beta_{q} .
\end{aligned}
$$

We now prove that (3.20) holds for $t_{m-t+1}=2$, and then it will follow, from Lemma 2.2, that it holds for all integers $t_{m-t+1}>2$. Hence putting $t_{m-t+1}=2$ in (3.20) and rearranging we obtain the inequality

$$
\begin{aligned}
& 4(q-1) \sum_{s=1}^{l-2} q^{l-1-s} k\left(\delta_{s}\right)+4 q \delta_{l-1}\left(\delta_{l-1}+3+\alpha_{q}-\beta_{q}+2 q^{-1}\right) \\
& +q^{2}-15 q+2\left(\beta_{q}-\alpha_{q}\right) q-4 \beta_{q}+20 \geqq 0
\end{aligned}
$$


Once again using the fact that $k\left(\delta_{s}\right) \geqq 0$ for all integers $\delta_{s}$, we are left with inequality (3.13), with $\delta_{1}$ replaced by $\delta_{l-1}$, whose validity has already been established. Thus it remains to consider the case when $t_{m-1+1}=1$, when (3.20) takes the form

$$
4(q-1) \sum_{s=1}^{l-2} q^{l-2-s} k\left(\delta_{s}\right)+4 \delta_{l-1}\left(\delta_{l-1}+5+\alpha_{q}-\beta_{q}\right)-3 q+9-2 \alpha_{q}-2 \beta_{q} \geqq 0 .
$$

We now have to run through the possible values of $l$, namely $3,4, \ldots, m$. Initially $l=3$, so that $m \geqq 3$, giving stage 3 , and (3.21) becomes

$$
4(q-1) k\left(\delta_{1}\right)+4 \delta_{2}\left(\delta_{2}+5+\alpha_{q}-\beta_{q}\right)-3 q+9-2 \alpha_{q}-2 \beta_{q} \geqq 0 .
$$

This splits into two cases.

(i) $3 \leqq \beta_{q}-\alpha_{q} \leqq 4$. For integral values of $\delta_{1}$, the least two values of $k\left(\delta_{1}\right)$ occur when $\delta_{1}=0$ and -1 , and $\min \left[k\left(\delta_{1}\right): \delta_{1} \in \mathbf{Z}-\{0,-1\}\right]=k(1)$. Also $\delta_{2}\left(\delta_{2}+5+\alpha_{q}-\beta_{q}\right)$ has a minimum value for integral $\delta_{2}$ when $\delta_{2}=-1$. We now show that (3.22) holds for all integral values of $\delta_{2}$ when $\delta_{1} \neq 0$ or -1 . This will follow from the validity of the inequality for $\left(\delta_{1}, \delta_{2}\right)=(1,-1)$. In this case, after some rearrangement, (3.22) becomes

$$
17 q-27-(4 q-10)\left(\beta_{q}-\alpha_{q}\right)-4 \beta_{q} \geqq 0 .
$$

As $\beta_{q}-\alpha_{q} \leqq 4$ it will be sufficient to verify that

$$
17 q-27-4(4 q-10)-4 \beta_{q} \geqq 0,
$$

that is

$$
4 \beta_{q} \leqq q+13
$$

As $\beta_{q} \leqq 3 q-\sqrt{ }\left(8 q^{2}-9 q+1\right)+2$, we have to verify that $11 q-5 \leqq 4 \sqrt{ }\left(8 q^{2}-9 q+1\right)$ or equivalently $q(7 q-34) \geqq 9$, which is obviously true for all $q \geqq 9$. Thus we are left with the cases $\delta_{1}=0$ or -1 .

(ii) $4<\beta_{q}-\alpha_{q} \leqq 5$. For integral values of $\delta_{1}$, the two least values of $k\left(\delta_{1}\right)$ occur when $\delta_{1}=0$ and 1 and $\min \left[k\left(\delta_{1}\right): \delta_{1} \in \mathbf{Z}-\{0,1\}\right]=k(-1)$. Also $\delta_{2}\left(\delta_{2}+5+\alpha_{q}-\beta_{q}\right)$ has a minimum value when $\delta_{2}=0$. We now test $(3.22)$ with $\left(\delta_{1}, \delta_{2}\right)=(-1,0)$ and obtain the inequality

$$
(4 q-2)\left(\beta_{q}-\alpha_{q}\right)-15 q+21-4 \beta_{q} \geqq 0 .
$$

As $\beta_{q}-\alpha_{q}>4$ it will suffice to verify that

$$
4(4 q-2)-15 q+21-4 \beta_{q} \geqq 0
$$

or equivalently

$$
4 \beta_{q} \leqq q+13
$$


which is (3.23) again. The cases $\delta_{1}=0$ or 1 remain.

If $l=m=3$ we have to consider the case when $t_{3}=t_{2}=t_{1}=1$ and $a_{3}=1$ together with $\delta_{1}=0$ or -1 when $3 \leqq \beta_{q}-\alpha_{q} \leqq 4$ or $\delta_{1}=0$ or 1 when $4<\beta_{q}-\alpha_{q} \leqq 5$. By (2.6), $T\left(q, n_{3}\right)=F_{3}(3)$ and so, with the usual substitutions for $a_{2}, a_{1}$ and $a_{0}$, it follows from Lemma 2.3 that the condition $T\left(q, n_{3}\right)>0$ is equivalent to

$$
4(q-1)\left\{q k\left(\delta_{1}\right)+k\left(\delta_{2}\right)\right\}+4 h\left(\delta_{3}\right)+q-7-2 \alpha_{q}+2 \beta_{q}>0,
$$

once again easily seen to be true for all integers $\delta_{1}, \delta_{2}$ and $\delta_{3}$.

Summing up, if $l=3$ the only cases which remain to be considered occur when

$$
t_{m}=t_{m-1}=t_{m-2}=1 \text { and } a_{m}=1
$$

together with

$$
\delta_{1}=\left\{\begin{array}{rrrl}
0 & \text { or } & -1 & \text { if } 3 \leqq \beta_{q}-\alpha_{q} \leqq 4 \\
0 & \text { or } & 1 & \text { if } 4<\beta_{q}-\alpha_{q} \leqq 5
\end{array}\right.
$$

We next take $l=4$, so that $m \geqq 4$, giving stage 4 , and then (3.21) becomes

$$
4(q-1)\left\{q k\left(\delta_{1}\right)+k\left(\delta_{2}\right)\right\}+4 \delta_{3}\left(\delta_{3}+5+\alpha_{q}-\beta_{q}\right)-3 q+9-2 \alpha_{q}-2 \beta_{q} \geqq 0 .
$$

When $\delta_{1}=0$ repetition of the argument at stage 3 leaves

$$
\delta_{2}=\left\{\begin{array}{llrl}
0 & \text { or } & -1 & \text { if } 3 \leqq \beta_{q}-\alpha_{q} \leqq 4 \\
0 & \text { or } & 1 & \text { if } 4<\beta_{q}-\alpha_{q} \leqq 5
\end{array}\right.
$$

When $\delta_{1}=-1$, so that $3 \leqq \beta_{q}-\alpha_{q} \leqq 4,(3.25)$ becomes

$$
4 q(q-1)\left(\beta_{q}-\alpha_{q}-3\right)-3 q+9-2 \alpha_{q}-2 \beta_{q}+4(q-1) k\left(\delta_{2}\right)+4 \delta_{3}\left(\delta_{3}+5+\alpha_{q}-\beta_{q}\right) \geqq 0 .
$$

In this case, $\min \left[k\left(\delta_{2}\right): \delta_{2} \in \mathbf{Z}-\{0,-1\}\right]=k(1)$ and $\delta_{3}\left(\delta_{3}+5+\alpha_{q}-\beta_{q}\right)$ has a minimum value when $\delta_{3}=-1$. Putting $\left(\delta_{2}, \delta_{3}\right)=(1,-1)$ in $(3.25)$ leads to the inequality

$$
4(q-1)^{2}\left(\beta_{q}-\alpha_{q}-3\right)+5 q-15+6\left(\beta_{q}-\alpha_{q}\right)-4 \beta_{q} \geqq 0,
$$

which is true. When $\delta_{1}=1$, so that $4<\beta_{q}-\alpha_{q} \leqq 5,(3.25)$ becomes

$4 q(q-1)\left(5+\alpha_{q}-\beta_{q}\right)-3 q+9-2 \alpha_{q}-2 \beta_{q}+4(q-1) k\left(\delta_{2}\right)+4 \delta_{3}\left(\delta_{3}+5+\alpha_{q}-\beta_{q}\right) \geqq 0$.

This time, for integral values of $\delta_{2}$, the two least values of $k\left(\delta_{2}\right)$ occur when $\delta_{2}=0$ or 1 and the $\min \left[k\left(\delta_{2}\right): \delta_{2} \in \mathbf{Z}-\{0,1\}\right]=k(-1)$. Also $\delta_{3}\left(\delta_{3}+5+\alpha_{q}-\beta_{q}\right)$ has a minimum value when $\delta_{3}=0$ so that putting $\left(\delta_{2}, \delta_{3}\right)=(-1,0)$ in (3.26) gives

$$
4(q-1)^{2}\left(5+\alpha_{q}-\beta_{q}\right)+5 q+1+2\left(\beta_{q}-\alpha_{q}\right)-4 \beta_{q} \geqq 0,
$$


which is true.

Thus if $l=4$ the only cases which remain to be considered occur when

$$
t_{m}=t_{m-1}=t_{m-2}=t_{m-3}=1 \text { and } a_{m}=1
$$

together with

$$
\left(\delta_{1}, \delta_{2}\right)=\left\{\begin{array}{lll}
(0,0),(0,-1),(-1,0) & \text { or }(-1,-1) & \text { if } 3 \leqq \beta_{q}-\alpha_{q} \leqq 4 \\
(0,0),(0,1),(1,0) & \text { or }(1,1) & \text { if } 4<\beta_{q}-\alpha_{q} \leqq 5
\end{array}\right.
$$

If $l=m=4$, we can assume that $t_{4}=t_{3}=t_{2}=t_{1}=1$ and $a_{4}=1$ together with condition (3.27). In this case, using Lemma 2.3 again, the condition $T\left(q, n_{4}\right)=F_{4}(4)>0$ is equivalent to

$$
4(q-1)\left\{q^{2} k\left(\delta_{1}\right)+q k\left(\delta_{2}\right)+k\left(\delta_{3}\right)\right\}+4 h\left(\delta_{4}\right)+q-7-2 \alpha_{q}+2 \beta_{q}>0
$$

which is true.

At stage 5 we have $l=5$, so that $m \geqq 5$. Then the inequality (3.21) takes the form

$$
4(q-1)\left\{q^{2} k\left(\delta_{1}\right)+q k\left(\delta_{2}\right)+k\left(\delta_{3}\right)\right\}+4 \delta_{4}\left(\delta_{4}+5+\alpha_{q}-\beta_{q}\right)-3 q+9-2 \alpha_{q}-2 \beta_{q} \geqq 0 .
$$

If $\delta_{1}=0,(3.28)$ is essentially the same as (3.25) with an obvious change of notation. Thus repetition of the argument at stage 4 leaves the following values of $\left(\delta_{2}, \delta_{3}\right)$ namely

$$
\left(\delta_{2}, \delta_{3}\right)=\left\{\begin{array}{lll}
(0,0),(0,-1),(-1,0) & \text { or }(-1,-1) & \text { if } 3 \leqq \beta_{q}-\alpha_{q} \leqq 4 \\
(0,0),(0,1),(1,0) & \text { or }(1,1) & \text { if } 4<\beta_{q}-\alpha_{q} \leqq 5
\end{array}\right.
$$

If $\delta_{1}=-1$, so that $3 \leqq \beta_{q}-\alpha_{q} \leqq 4,(3.28)$ becomes

$$
\begin{aligned}
& 4 q^{2}(q-1)\left(\beta_{q}-\alpha_{q}-3\right)-3 q+9-2 \alpha_{q}-2 \beta_{q} \\
& \quad+4(q-1)\left\{q k\left(\delta_{2}\right)+k\left(\delta_{3}\right)\right\}+4 \delta_{4}\left(\delta_{4}+5+\alpha_{q}-\beta_{q}\right) \geqq 0 .
\end{aligned}
$$

The left-hand side of (3.30) takes a minimum value when $\left(\delta_{2}, \delta_{3}, \delta_{4}\right)=(0,0,-1)$ and in this case the inequality becomes

$$
4 q^{2}(q-1)\left(\beta_{q}-\alpha_{q}-3\right)-3 q-7-6 \alpha_{q}+2 \beta_{q} \geqq 0 .
$$

If $8 q^{2}-9 q+1$ is not a perfect square, $\beta_{q}-\alpha_{q}-3>1 /(3 q)$ by (2.2), and (3.31) will be a consequence of

$$
4 q(q-1)-9 q-21-18 \alpha_{q}+6 \beta_{q}>0,
$$

or equivalently

$$
4 q^{2}-13 q-21+18\left(\beta_{q}-\alpha_{q}\right)-12 \beta_{q}>0
$$


As $\beta_{q}-\alpha_{q} \geqq 3$ it will suffice to show that

$$
4 q^{2}-25 q+33+12\left(q-\beta_{q}\right)>0
$$

which is easily true. If $8 q^{2}-9 q+1$ is a perfect square and $\beta_{q}-\alpha_{q}=3,(3.30)$ reduces to

$$
4(q-1)\left\{q k\left(\delta_{2}\right)+k\left(\delta_{3}\right)\right\}+4 \delta_{4}\left(\delta_{4}+5+\alpha_{q}-\beta_{q}\right)-3 q+9-2 \alpha_{q}-2 \beta_{q} \geqq 0,
$$

which is (3.25) again with an obvious change of notation. Hence the values of $\left(\delta_{2}, \delta_{3}\right)$ which remain are

$$
\left(\delta_{2}, \delta_{3}\right)=(0,0),(0,-1),(-1,0) \text { or }(-1,-1) .
$$

If $\delta_{1}=1$, so that $4<\beta_{q}-\alpha_{q} \leqq 5,(3.28)$ is

$$
\begin{aligned}
& 4 q^{2}(q-1)\left(5+\alpha_{q}-\beta_{q}\right)-3 q+9-2 \alpha_{q}-2 \beta_{q} \\
& \quad+4(q-1)\left\{q k\left(\delta_{2}\right)+k\left(\delta_{3}\right)\right\}+4 \delta_{4}\left(\delta_{4}+5+\alpha_{q}-\beta_{q}\right) \geqq 0 .
\end{aligned}
$$

The left-hand side of (3.33) takes a minimum value when $\left(\delta_{2}, \delta_{3}, \delta_{4}\right)=(0,0,0)$ and in this case the inequality becomes

$$
4 q^{2}(q-1)\left(5+\alpha_{q}-\beta_{q}\right)-3 q+9-2 \alpha_{q}-2 \beta_{q} \geqq 0 .
$$

If $8 q^{2}-9 q+1$ is not a perfect square, $5+\alpha_{q}-\beta_{q}>1 /(3 q)$ by (2.3), and consequently (3.34) follows provided that

$$
4 q^{2}-13 q+27-6\left(\alpha_{q}-\beta_{q}\right)-12 \beta_{q}>0 \text {. }
$$

As $\beta_{q}-\alpha_{q}>4$, it will suffice to show that

$$
q(4 q-25)+51+12\left(q-\beta_{q}\right)>0,
$$

which is true. On the other hand, if $8 q^{2}-9 q+1$ is a perfect square and $\beta_{q}-\alpha_{q}=5,(3.33)$ takes the form

$$
4(q-1)\left\{q k\left(\delta_{2}\right)+k\left(\delta_{3}\right)\right\}+4 \delta_{4}\left(\delta_{4}+5+\alpha_{q}-\beta_{q}\right)-3 q+9-2 \alpha_{q}-2 \beta_{q} \geqq 0 .
$$

Once again this is essentially the same as (3.25) and so the values of $\left(\delta_{2}, \delta_{3}\right)$ which remain are

$$
\left(\delta_{2}, \delta_{3}\right)=(0,0),(0,1),(1,0) \text { or }(1,1)
$$

In the special case when $l=m=5$ the condition $T\left(q, n_{5}\right)=F_{5}(5)>0$ is equivalent to

$$
4(q-1)\left\{q^{3} k\left(\delta_{1}\right)+q^{2} k\left(\delta_{2}\right)+q k\left(\delta_{3}\right)+k\left(\delta_{4}\right)\right\}+4 h\left(\delta_{5}\right)+q-7-2 \alpha_{q}+2 \beta_{q}>0,
$$


which is true.

Summing up stage 5 , we have proved that $T\left(q, n_{m}\right)>0$ except in the following cases:

$$
t_{m}=t_{m-1}=\cdots=t_{m-4}=1 \text { and } a_{m}=1
$$

together with $\delta_{1}=0$ and $\left(\delta_{2}, \delta_{3}\right)$ satisfying (3.29). In addition when $8 q^{2}-9 q+1$ is a perfect square

$$
\delta_{1}=-1 \text { and }\left(\delta_{2}, \delta_{3}\right) \in\{(0,0),(0,-1),(-1,0),(-1,-1)\} \text { when } 3 \leqq \beta_{q}-\alpha_{q} \leqq 4
$$

or

$$
\delta_{1}=1 \text { and }\left(\delta_{2}, \delta_{3}\right) \in\{(0,0),(0,1),(1,0),(1,1)\} \quad \text { when } 4<\beta_{q}-\alpha_{q} \leqq 5
$$

By now the critical form for $n_{m}$ is beginning to emerge. When $8 q^{2}-9 q+1$ is not a perfect square, at the end of stage $l$ for $5 \leqq l \leqq m$ we are left with the following situation:

$$
t_{m}=t_{m-1}=\cdots=t_{m-l+1}=1 \text { and } a_{m}=1
$$

together with

$$
\begin{aligned}
& \delta_{1}=\delta_{2}=\cdots=\delta_{l-4}=0 \text { and } \\
& \left(\delta_{l-3}, \delta_{l-2}\right)=\left\{\begin{array}{lll}
(0,0),(0,-1),(-1,0) & \text { or }(-1,-1) & \text { if } 3 \leqq \beta_{q}-\alpha_{q} \leqq 4 \\
(0,0),(0,1),(1,0) & \text { or }(1,1) & \text { if } 4<\beta_{q}-\alpha_{q} \leqq 5
\end{array}\right.
\end{aligned}
$$

In this case, by (2.5) and (3.17), we have

$$
T\left(q, n_{m}\right)=F_{m}(l-1)+n_{m-l} \sum_{r=m-l+1}^{m}\left[2 a_{r}-(q-1) t_{r}\right]+T\left(q, n_{m-l}\right) .
$$

Using the usual substitutions for $a_{r}(r=m-l+1, \ldots, m)$ together with $t_{m}=\cdots=t_{m-1+1}=1$ we obtain

$$
\sum_{r=m-l+1}^{m}\left[2 a_{r}-(q-1) t_{r}\right]=4-q-\beta_{q}+2 \delta_{l-1} .
$$

Then, application of Lemma 2.3 gives

$$
\begin{aligned}
T\left(q, n_{m}\right)= & \frac{1}{4} q^{t_{1}+\cdots+t_{m-l+1}}\left\{4(q-1)\left[q k\left(\delta_{l-3}\right)+k\left(\delta_{l-2}\right)\right]+4 h\left(\delta_{l-1}\right)+q-7-2 \alpha_{q}+2 \beta_{q}\right\} \\
& +\left[4-q-\beta_{q}+2 \delta_{l-1}\right] n_{m-l}+T\left(q, n_{m-l}\right) .
\end{aligned}
$$

Since $\delta_{1}, \ldots, \delta_{n}$ are all linearly bounded in terms of $q$ and $m$, it follows from (1.8) and (3.36) that

$$
\frac{S\left(q, n_{m}\right)}{n_{m}}+h_{q}=\frac{T\left(q, n_{m}\right)}{n_{m}} \rightarrow 0 \text { as } m \rightarrow \infty \text { if } m-l \text { remains fixed, }
$$


and this is the critical form mentioned earlier. If $l<m$ we proceed to stage $l+1$, and so on until $l=m$ when the process comes to a halt yielding no further critical cases. In particular, when $l=m$, at the end of stage $m$ we have to consider $T\left(q, n_{m}\right)$ subject to the conditions $t_{m}=t_{m-1}=\cdots=t_{1}=1$ and $a_{m}=1$ together with $\delta_{1}=\delta_{2}=\cdots=\delta_{m-4}=0$ and $\delta_{m-3}, \delta_{m-2} \in\{0,1,-1\}$. Using Lemma 2.3 gives

$$
\begin{aligned}
4 T\left(q, n_{m}\right)= & 4 F_{m}(m), \\
= & 4(q-1)\left\{q^{2} k\left(\delta_{m-3}\right)+q k\left(\delta_{m-2}\right)+k\left(\delta_{m-1}\right)\right\} \\
& +4 h\left(\delta_{m}\right)+q-7-2 \alpha_{q}+2 \beta_{q} .
\end{aligned}
$$

As we have seen $k(x) \geqq 0 \forall x \in Z$ and so

$$
4 T\left(q, n_{m}\right) \geqq 4 h\left(\delta_{m}\right)+q-7-2 \alpha_{q}+2 \beta_{q}
$$

$>0$

by the same argument as that towards the end of stage 1 .

When $8 q^{2}-9 q+1$ is a perfect square, it is clear that additional critical cases arise. In particular, when $\beta_{q}-\alpha_{q}=3$ we have $k(\delta)=\delta(\delta+1)$. In this case at the end of stage $l$ $(5 \leqq l \leqq m)$ we are left with

$$
\delta_{1}, \ldots, \delta_{l-4}, \delta_{l-3}, \delta_{l-2} \in\{0,-1\} .
$$

As $k\left(\delta_{i}\right)=0$ for $1 \leqq i \leqq l-2$ and $h\left(\delta_{l-1}\right)=\delta_{l-1}\left(\delta_{l-1}+3+\alpha_{q}-\beta_{q}\right)=\delta_{l-1}^{2}$ this leads to

$$
\begin{aligned}
T\left(q, n_{m}\right)= & \frac{1}{4} q^{t_{1}+\cdots+t_{m-1+1}}\left[4 \delta_{l-1}^{2}+q-1\right] \\
& +\left[4-q-\beta_{q}+2 \delta_{l-1}\right] n_{m-1}+T\left(q, n_{m-l}\right) .
\end{aligned}
$$

and once again

$$
\frac{S\left(q, n_{m}\right)}{n_{m}}+h_{q}=\frac{T\left(q, n_{m}\right)}{n_{m}} \rightarrow 0 \text { as } m \rightarrow \infty
$$

and $m-l$ remains constant. If $l<m$ we can proceed to the next stage as before. When $\beta_{q}-\alpha_{q}=5, k(\delta)=\delta(\delta-1)$ and the critical cases occur when $\delta_{1}, \ldots, \delta_{l-2} \in\{0,1\}$; otherwise the situation is similar.

November 1989. Since this paper was submitted for publication the analogous theorem for even values of $q$ has been obtained. A statement of the result and brief outline of the proof will follow.

\section{REFERENCES}

1. R. Bellman and H. N. Shapiro, On a problem in additive number theory, Ann. of Math. (2) 49 (1948), 333-340. 
2. L. E. Bush, An asymptotic formula for the average sums of the digits of integers, Amer. Math. Monthly 47 (1940), 154-156.

3. J. Coquet, Power sums of digital sums, J. Number Theory 22 (1986), 161-176.

4. H. Davenport, The Higher Arithmetic (Hutchinson's University Library, 1952).

5. H. Delange, Sur la fonction sommatoire de la fonction "somme des chiffres", Enseign. Math. 21 (1975), 31-47.

6. M. P. Drazin and J. S. Griffiths, On the decimal representation of integers, Proc. Cambridge Philos. Soc. 48 (1952), 555-565.

7. D. M. E. Foster, Estimates for a remainder term associated with the sum of digits function, Glasgow Math. J. 29 (1987), 109-129.

8. P. Kirschenhofer and R. F. Tichy, On the distribution of digits in Cantor representations of integers, $J$. Number Theory 18 (1984), 121-134.

9. G. LARCHER and R. F. TICHY, Some number-theoretical properties of generalized sum-ofdigits functions, Acta Arith. 52 (1989), 183-196.

10. M. D. McIlroy, The number of 1's in binary integers: bounds and extremal properties, SIAM J. Comput. 3 (1974), 255-261.

11. L. Mirsky, A theorem on representations of integers in the scale of $r$, Scripta Math. 15 (1949), 11-12.

12. I. Shiokawa, On a problem in additive number theory, Math. J. Okayama Univ. 16 (1974), $167-176$.

13. K. B. Stolarsky, Power and exponential sums of digital sums related to binomial coefficient parity, SIAM J. Appl. Math. 32 (1977), 717-730.

14. J. R. TRollope, An explicit expression for binary digital sums, Math. Mag. 41 (1968), 21-25.

15. J. R. Trollope, Generalized bases and digital sums, Amer. Math. Monthly 74 (1967), 690-694.

Mathematical Institute

UNIVERSITY OF ST ANDREWS

North Haugh

St ANDrews, KY16 9SS 